\title{
VATH Regimen
}

National Cancer Institute

\section{Source}

National Cancer Institute. VATH Regimen. NCI Thesaurus. Code C9638.

A chemotherapy regimen consisting of fluoxymesterone, thiotepa, doxorubicin, and vinblastine that may be used in the treatment of advanced breast cancer. 